\title{
How Parenting Anxiety, Number of Children, and Employment Status Affect the Parental Anger of Mothers with Young Children in Korea
}

\author{
Kai-Sook Chung ${ }^{1}$, Mina Kim² \\ Senior Research Fellow, Ddadeutan Educational Community Research Center, Pusan National University, Pusan, Korea ${ }^{1}$ \\ Assistant Professor, Soongeui Women's College, Seoul, Korea² \\ 유아기 자녀를 둔 어머니의 부모 분노: 양육불안, 자녀 수, 취업 여부의 영향 \\ 정계숙 ${ }^{1}$, 김미나 ${ }^{2}$ \\ 부산대학교 따뜻한교육공동체연구센터 전임연구원 ${ }^{1}$ 숭의여자대학교 조교수 ${ }^{2}$
}

Objectives: A difficulty in controlling anger causes dissonance and injury among members of society, and hence the healthy expression of anger by parents with young children is important to the community health of the future society. This study aimed to investigate relationships between parenting anxiety, the number of children in a family, maternal employment status, and the parental anger of mothers, in order to identify the main variables explaining parental anger.

Methods: The participants were 219 mothers of young children residing in Korea. The results of the Likert self-report by mothers were analyzed using descriptive, correlation, and multiple regression statistics.

Results: First, the more anxiety from parenting the mother experienced, the more anger as a parent they expressed. Also, the more children the mothers had, the higher levels of anger they experienced. Unemployed mothers experienced significantly more impulsive anger than their employed counterparts. Secondly, the number of children, maternal employment status, and three anxiety factors related to parenting efficacy, attachment with a child, and worries about a child explained parental anger.

Conclusion: Mothers with young children in Korea are more likely to pursue a united relationship between mother and child, suggesting that parenting anxiety, number of children, and maternal employment are important factors in the psychological wellbeing of Korean mothers of preschool children. Considering the importance of healthy mother-child relations and young children's learning of anger expression for the formation of a healthy community, support is critical to strengthen mothers' competence to manage parenting anxiety and anger expression.

Keywords: parental anger, parenting anxiety, number of children, employment status, mothers with young children

\section{Introduction}

Anger has been regarded as an emotional motivator that human beings develop when faced with hostility and aggression,

Corresponding Author: Mina Kim, Assistant Professor, Department of Early Childhood Education, SoongEui Women's College, Sopa-ro 2-gil, Jung-gu, Seoul, Korea

E-mail: mkim@sewc.ac.kr and individual differences in anger are seen as a personality trait (Spielberger, Jacobs, Russell, \& Crane, 1983). From the Darwinian view, anger is a spectrum of emotional states ranging from irritation or annoyance to fury or rage, and is characterized as an

(C)The Korean Association of Child Studies

This is an Open Access article distributed under the terms of the Creative Commons Attribution Non-Commercial License (http:// creativecommons.org/licenses/by-nc/4.0) which permits unrestricted noncommercial use, distribution, and reproduction in any medium, provided the original work is properly cited. 
adaptive mechanism for a human being to survive (Darwin, 1965). Meanwhile, the Freudian perspective focuses on the baneful aspect of anger, considering aggression as an instinctive drive motivating destructive behavior (Tavris, 1982). Anger has been regarded as a risky factor lowering the quality of one's life by not only leading to coronary heart disease or any other high blood pressure-related symptoms (Diamond, 1982) but also creating a distance between people if anger is not appropriately expressed or controlled. Based on a series of crimes and incidents related to anger control disorder in modern society, anger suitably expressed or suppressed seems to be an essential feature for a healthy community in addition to personal wellbeing (K.-S. Chung \& Kim, 2016).

Most research on parental anger has measured distress with inequality in parenting, reporting that women have higher levels of anger than men, that each additional child in the household increases anger, and that children cause more anger for mothers than fathers because of economic inequality and the inequitable distribution of parental responsibilities (Ross \& van Willigen, 1996). Parental anger is reported to have significant effects on child development including behavioral problems (Crnic, Gaze, \& Hoffman, 2005; Jeong \& Kim, 2014) and inconsistent parenting attitude (M. S. Kim \& Moon, 2005; Zahn-Waxler, Ianotti, Cummings, \& Denham, 1990). Young children facing direct parental anger are at high risk of being mentally overwelmed, which only hinders their subsequent socio-emotional development and mental health, especially in early childhood (Denham et al., 2000). Besides, when the anger expression is excessive, the parent develops neurotic and dysfunctional parenting behavior, thus heightening the likelihood of child abuse (Plate et al., 2019).

Based on society's material values, most Korean parents believe that academic achievement through fierce competition brings wealth and social success, and a strong sense of unity between parents and children directly connects their child's success to their own self-esteem (E. A. Choi, 2014; S. Choi, 1994). In the era of the Fourth Industrial Revolution, where earlier values and many professions are no longer significant, there is much discussion on the need for educational reform (D.-S. Kim, 2017). Today, parents may fear a new era that requires changes in their children's education, hence experiencing more anxiety about raising their children than previous generations of parents.

Anxiety is a feeling of conscious perception of tension or concern experienced by an individual in a stressful or threatening situation (Spielberger, 1972). From the clinical view, anxiety is a cognitive, affective, physiological, and behavioral reaction to aversive or unexpected circumstances, and it is followed by the perception of unavoidable or uncontrollable situations (Clark \& Beck, 2010). Barlow (2002) defined anxiety as a perception of unpredictability over potential danger, which equips a person to prepare for the future in comparison to fear, which is an alarm to current danger. Frustration and helplessness can follow anxiety, making people feel powerless to control their autonomy. Anxiety includes cognitions of harm and danger (Beck, Brown, Steer, Eidelson, \& Riskind, 1987), as well as aggressiveness related cognitions including suspiciousness and expectation that others will hurt, abuse, lie, or take intentional advantage (Calvete, Estévez, López de Arroyabe, \& Ruiz, 2005). As anxiety leads to a feeling of helplessness, anger makes them feel better because it helps them repossess control (Bright, 1996).

With an understanding that anger is a natural and rational expression, parenting anxiety is an emotional experience that occurs when parents experience difficulties related to parenting throughout the life cycle of a family and the growth of their children. In particular, parenting anxiety is a major source of parenting stress for parents of infants and toddlers. Daily parenting stress can lead to negative emotions such as depression and anger in mothers with young children (K.-S. Chung, Cha, \& Kim, 2019; E.-K. Kim \& Koh, 2016). Inappropriate parenting experience not only impedes children's socio-emotional development in their early developmental stages but also increases the possibility of child behavioral problems, or even child abuse depending on the degree of anger (Rodriguez \& Green, 1997). Previous research on parental anxiety and anger has mainly targeted children with disabilities and their parents (Rezendes \& Scarpa, 2011), and there are insufficient empirical studies on the expression of anger due to inadequate parenting experience or parenting anxiety. Furthermore, studies on mothers of young children in normal families are particularly limited (K.-S. Chung, 2019; E.-K. Kim \& Koh, 2016).

Anxiety exists in all cultures but the subjective experiences are differently shaped by culture-specific factors (Barlow, 2002). Most importantly, the standard diagnostic classification system is based on the Western conceptualizations (De Jong \& van Ommeren, 2002), and thus may not have a high validity in other cultures. Since anger is an affect constructed by society and socialized 
through cultural paradigms (Averil, 1982), parental anger may reflect different aspects depending on the culture. Korean parents today are more interested in child rearing than their parents and want to be helped in various ways. At the same time, the vast amount of information about parenting through mass media or experts is causing confusion and anxiety (We \& Chae, 2015). Most mothers in Korea today need to raise their children alone without the help of relatives due to the increase of nuclear families. As such, they are more obsessed about leading their children to a successful life, while juggling the many roles expected of them by the Confucian patriarchal family structure. Most Korean parents believe that high academic achievement through intense competition from preschool leads to economic and social success.

Accordingly, as the social context of child education has become more competitive, mothers' support for the entire process of child education as well as child rearing became very important (H. K. Park, 2009). The Korean language uses, the term 'school parent' along with 'parent' to specifically refer to the parents of students. From kindergarten to college, all documents distributed by the institutions are addressed to 'school parents' rather than just 'parents'. This also makes parents feel as if they have new roles. In the transition from preschool mothers to elementary school parents, 'school parent networking', 'family (children and spouses)', and 'private education institutions' assisted mothers in learning their new roles. These factors allowed mothers to exchange educational information, observe and compare their support activities with other parents, and actively search for information to help in decision-making through the school-parent network (M. Kang, 2017). Even with young children, Korean mothers are thus burdened and anxious about adhering to success-oriented parenting (We \& Chae, 2015).

Korea is currently facing lowest-low fertility, which is a social and economic problem, exacerbated by the improvement of women's education levels, social participation, delayed marriage, desire for self-realization, and employment. In 2018, Korea's total fertility rate was only .98 , marking it the only country to have a fertility rate under 1 . The number even falls below the lowest-low fertility rate standard of 1.3 , as well as being far from the average of 1.68 in OECD advanced countries (Statistics Korea, 2019). For parents who believe that high academic achievement is directly related to their children's future success in the midst of rapid global change, expectations towards children are heightened, particularly as the frequency of childbirth is gradually decreasing to about one per family (Shin, Kee, Woo, \& Yoon, 2014). If they have fewer children, they tend to become excessive child rearing and education for their children (Byeon, 2010). This further increases parenting anxiety and can lead to frequent experiences of negative emotions such as anger or depression. On the other hand, older research has shown that the more children mothers have, the less likely they are able to give adequate attention and affection to due to time and role constraints, as well as related psychological and financial burdens, possibly causing future problems, including the children's rejetion of their mothers (Lavee, Sharlin, \& Katz, 1996). As M. Kang (2017) pointed out, it is clear that the role of mothers in creating learning conditions for their children after elementary school will become more complicated as the number of children increases. This will also increase the parenting burden and anxiety, and can cause parents to frequently experience negative emotions such as anger or depression. While this suggests that a mother's upbringing of her children can differ depending on the number of children she has, there is no agreement on this in the literature. Despite conflicting findings however, it is clear that the number of children affect parents' anxiety and anger.

Intensive care in early childhood is a decisive factor in child development (Bornstein, 2002). Employment is the most common factor preventing mothers from providing such intensive care. According to some research findings, maternal employment is not significantly related to mothers' parenting behavior (J.-H. Kim \& Moon, 2006) or parenting efficacy (G. H. Kim, 2011). In general, however, mothers who feel helpless put less effort into their relationships with their children. They also tend to lack consistency in parenting and nurturing characterized by rejection and punishment, rather than warmth and acceptance (Crnic \& Greenberg, 1990; Huth-Bocks, Guyon-Harris, Calvert, Scott, \& Ahlfs-Dunn, 2016). The inconsistency in parenting behavior caused by such a sense of helplessness is highly likely to cause parenting anxiety or stress related to self-confidence as a parent (K.S. Chung \& Kim, 2014). Working mothers have a higher level of parenting stress than non-working mothers due to the roleoverload in balancing work and child care (Adhikari, 2012). On the other hand, non-employed mothers experience more parenting stress because they spend more time with their children, and are 
more involved in their care than working mothers (Lee \& Shin, 2013; Sohn, 2012) While research shows different results regarding parenting stress behavior depending on whether or not they are employed, there is agreement that both working and non-working mothers are excessively burdened as mothers in different aspects, and that they are hence more likely to experience negative emotions such as anger due to performing various roles.

As seen above, parenting stress variables such as parenting anxiety specifiacally affect mothers with young children, while sociocultural specificity and demographic variables such as the number of children and employment status have an effect on mothers' expression of anger in the parenting process. However, there are few studies investigating the relationship of mothers and young children in a typical developmental trajectory. In particular, few studies have compared the magnitude of the influence between the two variables. Therefore, this study aims to investigate the relative explanatory power of parenting anxiety variables, the number of children, and mothers' employment status on the expression of parental anger. The research questions are as follows:

1. What is the relationship between parenting anxiety, the number of children, employment status, and parental anger of mothers with young children?

2. What is the relative explanatory power of parenting anxiety, the number of children, and employment status in prediction of parental anger of mothers with young children?

\section{Methods}

\section{Procedures and Participants}

The study participants were 219 mothers with young children residing in metropolitan areas of Korea. The questionnaires were distributed to two daycare centers and two kindergartens in the areas. The authors provided sufficient explanations about the purposes and contents of the study and informed consent was obtained from each participant through the teachers. Participants were aged from 27 to 46 years, and $52 \%$ of the mothers in their late 30 s, $32 \%$ in their early 30 s, $15 \%$ in their 40 s, and $2 \%$ in their late 20 s. Regarding the mothers' education, $88 \%$ of the participants were graduates of universities and above, and 57\% were employed.
With respect to the number of children, $61 \%$ of the mothers had two children, $28 \%$ had one child, and $11 \%$ had three children. The children's ages ranged from 3 to 6 and $51 \%$ were girls.

\section{Measurements}

\section{Parental Anger}

Parental anger was assessed using PAS-K (K.-S. Chung, Son, Ryu, \& Cha, 2016), the Korean adaptation of the Parent Anger Scale (H. J. Park, 2017). The PAS-K, a 6-point Likert scale ranging from 30 to 180 points, has some modifications to the PAS items with consideration of parenting situations and experiences in Korea for the mothers of young children. In addition, the scale was developed to measure parental anger in Korea, where mothers find it difficult to respond to some questions due to feelings of guilt or shame at expressing anger. The total of 18 items on the PAS-K were grouped into three areas: situational anger experience (10 items), impulsive behavior ( 5 items), and anger management difficulty (3 items). The internal consistency of PAS-K in this study was fairly good having Cronbach's alphas for situational anger experience, impulsive behavior, anger management difficulty, and overall as .89, .79, .65, and .93 , respectively.

\section{Parenting Anxiety}

Mothers' parenting anxiety was assessed using the Parenting Anxiety Scale (PAS) and the scale was developed by We and Chae (2015), based on literatures (Abidin, 1992; Belsky, 1984; Lazarus \& Averill, 1972). The PAS consists of 26 items grouped into five subscales: anxiety related to parental role efficacy (six items), anxiety related to attachment with a child (five items), anxiety related to worries about a child (five items), anxiety related to social support (four items), and anxiety related to perfectionism (six items). Mothers rated themselves on the 5-point Likert scale (never [1] - very much [5]). A higher score in each sub-scale reflects the experience of a higher level of anxiety from parenting. The Cronbach's alphas in the study were .86 for anxiety related to parental role efficacy, .84 for anxiety related to attachment with a child, .77 for anxiety related to worries about a child, .68 for anxiety related to social support, and .80 for anxiety related to perfectionism. 


\section{Statistical Analyses}

Depending on the research questions, the mean and standard deviation, Pearson correlation, regression analyses were conducted using SPSS 23.0 (IBM Co., Armonk, NY). Stepwise multiple regression analyses were used to test the relative explanatory power of each predicting variables on parental anger. The number of children and employment status were entered in step 1, and parenting anxiety were entered in step 2. Listwise deletion was performed for missing values.

\section{Results}

\section{Relationships between parenting anxiety, number of children, employment status and parental anger}

Data in parental anger, parenting anxiety, number of children, and dummy-coded maternal employment status factors were normally distributed in the datasets (i.e., skewness $<3$, kurtosis $<$ 10). Mothers perceived their anger management difficulty as low compared to their impulsive and situational anger as a parent. Mothers experienced fairly high anxiety from parenting, with anxiety related to efficacy and anxiety related to worries about a child being relatively higher than anxieties related to perfectionism, social support, and attachment as shown in Table 1.

Three parental anger factors (i.e., anger management difficulty, impulsive anger, and situational anger) were positively correlated with all parenting anxiety factors (i.e., anxieties related to parenting efficacy, attachment between mothers and children, worries about their children, social support, and perfectionism of mothers) at a significatly high level $(.20<r s<.62$, ps $<.001)$ as shown in Table 1 . The more anxiety mothers perceived from parenting, the more anger they expressed as parents, or vice versa. In particular, mothers who felt their anxiety from worries about a child were found to have more anger or vice versa. The number of children mothers had was positively correlated with parental anger factors except anger management difficulty. The more children mothers had, the more they felt impulsive anger, overall anger, and situational anger in that order, or vice versa. Depending on their employment status, mothers experienced parenting anxiety and parental anger at different levels. Unemployed mothers significantly experienced more of impulsive parental anger than their employed counterparts. Overall, parenting anxiety and parental anger are positively correlated, and the correlation is relatively higher between anxiety related to worries about a child and parental anger.

Table 1

Descriptive Statistics and Correlations among Parental Anger, Number of Children, Employment Status, and Parenting Anxiety of Mothers

\begin{tabular}{|c|c|c|c|c|c|c|c|c|c|c|c|}
\hline Variables & 1 & 2 & 3 & 4 & 5 & 6 & 7 & 8 & 9 & 10 & 11 \\
\hline 1. Parental anger-management difficulty & - & & & & & & & & & & \\
\hline 2. Parental anger-impulsive & $.81^{* * *}$ & - & & & & & & & & & \\
\hline 4. Parental anger total & $.85^{* * *}$ & $.93^{* * *}$ & $.97^{* * *}$ & - & & & & & & & \\
\hline 5. Parenting anxiety-efficacy & $.45^{* * *}$ & $.45^{* * *}$ & $.57^{* * *}$ & $.55^{* * *}$ & - & & & & & & \\
\hline 6. Parenting anxiety-attachment & $.39^{* * *}$ & $.44^{* * *}$ & $.57^{* * *}$ & $.54^{* * *}$ & $.63^{* * *}$ & - & & & & & \\
\hline 8. Parenting anxiety-support & $.20^{* * *}$ & $.29^{* * *}$ & $.41^{* * *}$ & $.36^{* * *}$ & $.56^{* * *}$ & $.65^{* * *}$ & $.62^{* * *}$ & - & & & \\
\hline 9. Parenting anxiety-perfectionism & $.28^{* * *}$ & $.30^{* * *}$ & $.45^{* * *}$ & $.41^{* * *}$ & $.58^{* * *}$ & $.47^{* * *}$ & $.69^{* * *}$ & $.57^{* * *}$ & - & & \\
\hline 10. Number of children & .10 & $.12^{*}$ & $.18^{* *}$ & $.16^{* *}$ & .04 & .09 & -.00 & -.01 & .08 & - & \\
\hline 11. Employment status_dummy & $-.12^{*}$ & $-.17^{* *}$ & -.08 & $-.12^{*}$ & .01 & .04 & -.05 & .02 & .05 & -.06 & - \\
\hline$M$ & 2.05 & 2.45 & 2.59 & 2.46 & 2.53 & 1.75 & 2.38 & 1.88 & 2.19 & 1.83 & .57 \\
\hline
\end{tabular}

Note. $N=218$. Employment was coded using dummy coding (not employed $=0$, employed $=1$ ).

${ }^{*} p .05 .{ }^{* *} p<.01 .{ }^{* * *} p<.001$. 
Prediction of parental anger by parenting anxiety, number of children, and employment status of mothers

The result of diagnosing multicollinearity that examined the correlation between variables in performing multiple regression analyses indicated no multicollinearity with tolerances above .1 and VIFs (Variances Inflation Factors) below 10. Stepwise multiple regression analyses were performed using parenting anxiety, number of children, and employment status of mother as predictor variables, and each of three sub-factors of parental anger and overall parent anger as dependent variables in four separate regression analyses as shown in Table 2. Parental anger overall was explained by the number of children and employment in step 1 with $3 \%$ of explanatory power and the model was significant $(F=$ $4.16, p<.05)$. In step 2 , the five parenting anxiety variables were added, and the total explanatory power increased to $44 \%$. In terms of the magnitude of explanatory power, it appeared to be in the order of anxiety related to worries about children, anxiety related to attachment, anxiety related to efficacy, and number of children, in a positive way and employment status in a negative way, and the model was significant $(F=24.04, p<.001)$. Therefore, it can be seen that parenting anxiety has a relatively greater explanatory power for parental anger overall than the number of children or the employment status of mothers, and among the factors of parenting anxiety, worries about a child, attachment, and parenting efficacy are significant factors.

Impulsive anger, in the first step, was explained by the number of children and employment status significantly with $4 \%$ of explanatory power, and the model was significant $(F=4.49, p<$ $.05)$. In step 2 , the total explanatory power improved to $32 \%$, and showed significant explanatory power in the order of anxiety related worries about a child, attachment anxiety, and employment status, and the model was significant $(F=17.67, p<.001)$. It was found that parenting anxiety has a relatively greater explanatory power on the impulsive anger of parents than the employment status, and among the factors of parenting anxiety, worries about a child and attachment have a positive effect, while employment status has a negative effect.

Situational anger was initially explained by the number of children and employment status with $3 \%$ of explanatory power suggesting a significant model $(F=4.03, p<.05)$. The total

Table 2

Multiple Regressions on Parental Anger using Number of Children, Employment Status, Parenting Anxiety in Mothers

\begin{tabular}{|c|c|c|c|c|c|c|c|c|}
\hline \multirow[t]{2}{*}{ Variables } & \multicolumn{2}{|c|}{$\begin{array}{l}\text { Parental anger } \\
\text { total }\end{array}$} & \multicolumn{2}{|c|}{$\begin{array}{l}\text { Parental anger } \\
\text {-impulsive }\end{array}$} & \multicolumn{2}{|c|}{$\begin{array}{l}\text { Parental anger } \\
\text {-situational }\end{array}$} & \multicolumn{2}{|c|}{$\begin{array}{c}\text { Parental anger } \\
\text {-management difficulty }\end{array}$} \\
\hline & $B(S E)$ & $\beta$ & $B(S E)$ & $\beta$ & $B(S E)$ & $\beta$ & $B(S E)$ & $\beta$ \\
\hline \multicolumn{9}{|l|}{ (Constant) } \\
\hline Number of children & $.21(.09)$ & $.15^{*}$ & $.17(.10)$ & .12 & $.26(.10)$ & $.18^{* *}$ & $1.33(1.0)$ & .09 \\
\hline Employment_dummy & $-.18(.11)$ & -.11 & $-.29(.12)$ & $-.16^{*}$ & $-.12(.12)$ & -.07 & $-1.96(.12)$ & .12 \\
\hline$\overline{R^{2}}$ & \multicolumn{2}{|c|}{$.03^{*}$} & \multicolumn{2}{|c|}{$.04^{*}$} & \multicolumn{2}{|c|}{$.03^{*}$} & \multicolumn{2}{|c|}{.02} \\
\hline$F$ & \multicolumn{2}{|c|}{$4.16^{*}$} & \multicolumn{2}{|c|}{$4.49^{*}$} & \multicolumn{2}{|c|}{$4.03^{*}$} & \multicolumn{2}{|c|}{2.48} \\
\hline \multicolumn{9}{|l|}{ (Constant) } \\
\hline Number of children & $.18(.07)$ & $.13^{*}$ & $.14(.09)$ & .09 & $.21(.08)$ & $.17^{* *}$ & $.09(.08)$ & .07 \\
\hline Employment_dummy & $-.18(.08)$ & $-.11^{*}$ & $-.29(.11)$ & $-.14^{*}$ & $-.12(.09)$ & -.07 & $-.19(.10)$ & -.11 \\
\hline PA-efficacy & $.18(.09)$ & $.17^{*}$ & $.17(.11)$ & .15 & $.17(.09)$ & $.16^{*}$ & $.24(.10)$ & $.23^{*}$ \\
\hline PA-attachment & $.34(.10)$ & $.28^{* *}$ & $.33(.12)$ & $.25^{* *}$ & $.37(.10)$ & $.28^{* * *}$ & $.28(.11)$ & $.22^{*}$ \\
\hline PA-worry & $.41(.11)$ & $.35^{* * *}$ & $.45(.14)$ & $.35^{* *}$ & $.41(.12)$ & $.33^{* *}$ & $.36(.13)$ & $.29^{* *}$ \\
\hline PA-support & $-.18(.10)$ & -.13 & $-.20(.12)$ & -.13 & $-.14(.11)$ & -.10 & $-.34(.12)$ & $-.24^{* *}$ \\
\hline PA-perfectionism & $.01(.10)$ & -.01 & $-.04(.12)$ & -.03 & $.05(.10)$ & .05 & $-.02(.11)$ & -.02 \\
\hline$R^{2}$ & \multicolumn{2}{|c|}{$.44^{* * *}$} & \multicolumn{2}{|c|}{$.32^{* * *}$} & \multicolumn{2}{|c|}{$.47^{* * *}$} & \multicolumn{2}{|c|}{$.29^{* * *}$} \\
\hline$\triangle R^{2}$ & \multicolumn{2}{|c|}{.41} & \multicolumn{2}{|c|}{.28} & \multicolumn{2}{|c|}{.44} & \multicolumn{2}{|c|}{.27} \\
\hline$F$ & \multicolumn{2}{|c|}{$24.04^{* * *}$} & \multicolumn{2}{|c|}{$17.67^{* * *}$} & \multicolumn{2}{|c|}{$27.11^{* * *}$} & \multicolumn{2}{|c|}{$15.64^{* * *}$} \\
\hline
\end{tabular}

Note. $N=218$. Employment was coded using dummy coding (not employed $=0$, employed $=1$ ).

${ }^{*} p<.05 .{ }^{* *} p<.01 .{ }^{* * *} p<.001$. 
explanatory power in the next step increased to $47 \%$ indicating a significant model $(F=27.11, p<.001)$. Regarding the magnitude in prediction power, anxiety related to worries about a child explained situational anger the most, followed by anxiety related to attachment, number of children, and anxiety related to efficacy. Therefore, it can be seen that parenting anxiety has a relatively greater explanatory power for the situational anger of parents compared to the number of children, and among the factors of parenting anxiety, worries about a child, attachment, and parenting efficacy are significant factors.

Anger management difficulty was not explained by the number of children and employment status in the first step, however, the explanatory power was $29 \%$, and having parenting anxiety variables added in the final step indicated a significant model $(F=$ $15.64, p<.001)$. Anxiety related to worries about a child explained parents' anger management difficulty the most, followed by anxiety of support, anxiety related to efficacy, and anxiety related to attachment. Therefore, it can be seen that parenting anxiety has a relatively greater explanatory power for anger management difficulty compared to demographic variables. Among the factors of parenting anxiety, anxieties related to worries about a child, attachment, and efficacy had a positive effect while anxiety related to support had a negative effect on parental anger management difficulty.

\section{Discussion}

Despite the significance of parental anger among mothers of young children, there has been little effort to identify its characteristics within the relationship with endogenous and exogenous factors affecting parental anger simultaneously. This study aimed to investigate how much parental anger is explained by parenting anxiety as an endogenous factor and by the number of children and maternal employment status as exogenous factors.

First of all, the current study confirmed that parental anger was significantly associated with anxiety from parenting in support of previous findings (K.-S. Chung et al., 2019; E.-K. Kim \& Koh, 2016). In particular, in terms of the magnitude of association with parental anger, parenting anxiety related to worries about a child was the greatest among all anxiety factors. This is similar to the findings of a study comparing the relative influence of parental competence and parenting anxiety on parental anger (K.-S. Chung, 2019). These results imply that mothers' excessive concern about the appropriateness of their child's behavior and the evaluation of others in Korean society can be easily expressed as anger under different parenting conditions, such as internal factors including parental competence or demographic factors. With no relevant research findings yet, it is assumed that this might be attributed to the Korean socio-cultural characteristics that emphasize the evaluation of others, and lead Korean parents to identify the accomplishments and success of their children as their own due to the sense of parent-child unity (S. Choi, 1994). This is not unique to Korean culture but typical in eastern countries in terms of emphasizing instrumental support and parental sacrifices in parenting (Wu \& Chao, 2017). Therefore, it is necessary to strengthen parenting competence, which is known to act as a protective factor to suppress anger (K.-S. Chung et al., 2019), Parenting competence can remove feelings of being unable to support their children as much as other parents, which feelings in turn can cause frustration and anger.

Positive associations between parental anger and the number of children can be explained by the role scarcity hypothesis (Repetti, Matthews, \& Waldron, 1989). According to the hypothesis, a mother with more children is required to perform multiple functions (e.g., preparing regular meals, feeding a baby, helping with homework) simultaneously, resulting in insufficient energy. At this point, inter-role-conflicts occur, roles collide, and the mother cannot perform any role properly. The daily routine with younger children particularly requires a lot of physical attention, time, and energy from the mother, so they have less free time than parents of older children. This limited free time may place a burden on the mothers' psychological wellbeing.

Parental anger, meanwhile, was associated with the employment status of mothers negatively, that is, employed mothers had less parental anger than unemployed mothers. This result is contrary to previous findings that working mothers feel guilty for not being able to adequately play the role of parents due to the overload of work and childcare (J.-N. Chung, 2016; S.-S. Park, 2004) while supporting studies reporting higher parenting stress among unemployed mothers (Kwon, 2011; Yoon, Hwang, \& Cho, 2009). This can be attributed to the responsibility, social isolation, physical 
fatigue, and financial burden experienced among unemployed mothers who are fully responsible for raising their children. Compared to working mothers, non-working mothers have a higher burden of private education and child support, and they suffer from parenting and housework significantly on weekends, while receiving less support from their husbands. These factors can lead to parental anger. On the other hand, in the case of working mothers, it can be seen that they experience less burden from parenting itself, because they are not directly in charge of raising children. Furthermore, improved parenting conditions, as well as more flexible working hours for working mothers in Korea, compared to the past, can be inferred as lowering their stress levels. Therefore, while investigating the relationship between parental anger and maternal employment status, it is necessary to also conduct follow-up studies examining fathers' roles in parenting aside from the effects of mothers' working conditions.

Secondly, parenting anxiety, number of children, and employment status were significant explanatory variables for overall parental anger, while differing depending on sub-factors of parental anger. In terms of overall parental anger, anxiety related to worries about a child, anxiety related to attachment, and anxiety related to parenting efficacy were significant predictors as well as the number of children. This suggests that these three anxiety factors are more directly connected to the parent-child relationship than the other two anxiety factors (i.e., anxiety related to social support and anxiety of perfectionism), which are more extrinsic (i.e., contextual) and intrinsic (i.e., personal characteristic), respectively. The finding that employment status has become a significant predictor of parental anger along with parenting anxiety suggests that these two variables may have an interactive effect. It is necessary to confirm this in subsequent studies.

Impulsive anger was explained by the anxiety of worries about a child, and attachment-related anxiety positively, and maternal employment negatively. Interestingly, maternal employment played a role as a predictor only on impulsive anger among all three anger sub-factors. This finding supports neither the previous findings of no significant relevance between maternal employment and negative emotional experience as parents (Adhikari, 2012; G. H. Kim, 2011; J.-H. Kim \& Moon, 2006), nor studies reporting significant effects of maternal employment on undesirable parenting attitude, or the unstable psychological state of mothers
(Crnic \& Greenberg, 1990; Huth-Bocks et al., 2016; Lee \& Shin, 2013; Sohn, 2012). One possible reason for the lower impulsive anger among working mothers is that their self-regulation ability improved through their many interpersonal experiences from their employment. Furthermore, job satisfaction is known to moderate the relationship between employment status and parental anger (K.W. Kim, Doh, Kim, \& Rhee, 2010). This and other employement related variables can be considered in a follow-up study.

Situational anger was explained by the anxiety of worries about a child, attachment-related anxiety, parenting efficacy related anxiety, and the number of children. The number of children, interestingly, played a role as a predictor only on situational anger among all three anger sub-factors. Mothers with more children may express situational anger more often than mothers with fewer children since they lack the absolute hours spent in parenting. The daily schedule for infants and young children are intense, repetitive activities that require intensive physical labor related to physiological needs such as eating, sleeping, changing diapers, washing, and so on. Thus, as the number of children increases, the amount of time consumed increases proportionally, while energy consumption increases exponentially. Accordingly, the amount of time for mothers to rest or to have time for themselves is reduced inversely. The situational peculiarity of mothering infants and preschool children in the Korean culture, which values the unique role of mothers in caring for young children (Kang, 2015) could lead these mothers to express more anger than ordinary adults or mothers with older children. As explained in the frustrationaggression hypothesis, it seems that situational anger can occur due to frustration or guilt at not being able to take care of a child properly, due to exhaustion or parental burnout. To understand the mechanism among situational anger, the number of children, and parental exhaustion, close observation or clinical studies are needed in the future.

Anger management difficulty was explained by predictor variables in different patterns from those of other parental anger sub-factors. Unexpectedly, the number of children and employment status were insignificant whereas anxiety related to support explained anger management difficulty only among all three anger sub-factors. From a cognitive neuroscience perspective, anger is the reaction of the human body's perceiving of a threat (Blair, 2012). Therefore, having difficulty controlling anger means having a hard 
time in controlling one's self response to threatening events or targets, that is, tending to express anger easily. Since this is a traitlike characteristic, it can be seen as a wife's tendency to perceive the husband's support in parenting as sufficient or insufficient, which is less affected by external factors such as the number of children or employment status. The result indicates that when mothers have no assistance in parenting or have higher anxiety due to the lack of trust in the assistance, anger management is lowered. This is in contrast to previous findings that social support will counteract the adverse effects of parenting stress and lead to better parenting (Ceballo \& Mcloyd, 2002) and that insufficient support or solutions from neighbors have a more serious effect on the psychological adjustment and wellbeing of parents (E.-K. Kim \& Koh, 2016). It could be said that the absence of reliable parenting support and the anxiety resulting from that might have different functions in positive parenting behavior. The lack of social support may, in a conflicted parenting situation, cause the mother to be more cautious and careful about her negative emotional control. Humans are social beings, and choose their actions by being conscious of others' reactions, and sometimes avoid others' evaluation due to fear. However, an appropriate level of anxiety can have a positive effect that leads to better achievement $(H$. Park \& Park, 2015). The average anxiety related to social support was 1.88 which was less than 'somewhat' as shown in Table 1. The influence of social support for parenting and anxiety related to support on parental anger needs to be studied in more depth.

In conclusion, parenting anxiety, number of children, and employment status were the influential factors for the parental anger of parents of young children, and parenting anxiety was more powerful than the other two variables. The results of the study are meaningful to understand the anger of mothers with young children and to serve as fundamental data for the development of programs to improve mothers' competence as caregivers. In addition, this study has various implications for a government policy respondinng to lowest-low birth rates. While there is no simple method to increase the number of children families wish to have, sufficient support related to parenting should be given in families with more children. Furthermore, a social atmosphere of 'co-parenting' should be created, where couples collaboratively parent their children rather than depending on the single parenting provided by mothers.
Modern society has suffered from frequent societal incidents due to so-called "anger control disorder." In order for young children to learn how to desirably express their anger, leading to a healthy community, it is important for the expression of parental anger to be properly managed. This study confirmed the effect of parenting anxiety on parental anger in Korean society, where the unity of the parent-child relationship is the main parenting value. The study also reveals the influence of the number of children and maternal employment, both of which are major social issues, and indicates certain variables that need more research and attention to manage parental anger. At the same time, this study has its limitations, such as the study variables being measured by self-reported questionnaires. Multiple methods including observation and interviews of other family members for assessing anxiety and anger management, as well as additional measurements of demographics and other individual variables (e.g., trait-anger, stress or satisfaction from work, relationship, spousal parenting) would enhance the interpretation of the study hypotheses. Second, participating mothers all belonged to the socioeconomic middle class, making the sample unrepresentative. Future studies should therefore recruit mothers from broad socioeconomic backgrounds. Furthermore, future studies need to examine the processes that lead to the wellbeing of individuals using longitudinal data in pursuit of cultural diversity in samples.

\section{Acknowledgements}

This study was supported by the National Research Foundation of Korea Government (NRF-2017S1A3A2067778).

\section{Notes}

This article was presented at 2020 Spring Online Conference of Korean Association of Child Studies.

\section{Conflict of Interest}

No potential conflict of interest relevant to this article was reported. 


\section{References}

\section{In English}

Abidin, R. R. (1992). The determinants of parenting behavior. Journal of Clinical Psychology, 21(4), 407-412. doi:10.1207/ s15374424jccp2104_12

Adhikari, H. (2012). Anxiety and depression: Comparative study between working and non-working mothers. Global Journal of Human-Social Science, 12(12), 1-9.

Averil, J. (1982). Anger and aggression: An essay on emotion. New York: Springer-Verlag.

Barlow, D. H. (2002). Anxiety and its disorders: The nature and treatment of anxiety and panic (2nd ed.). New York: Guilford Press.

Beck, A. T., Brown, G., Steer, R. A., Eidelson, J. I., \& Riskind, J. (1987). Differentiating anxiety and depression: A test of the cognitive content-specificity hypothesis. Journal of Abnormal Psychology, 96(3), 179-183. doi:10.1037/0021843X.96.3.179

Belsky, J. (1984). The determinants of parenting: A process model. Child Development, 55(1), 83-96. doi:10.2307/1129836

Blair, R. J. R. (2012). Considering anger from a cognitive neuroscience perspective. WIREs Cognitive Science, 3(1), 65-74. doi:10.1002/wcs.154

Bornstein, M. H. (2002). Parenting infants. In Bornstein, M. H. (Ed.), Handbook of parenting: Children and parenting (Vol. 1, pp. 3-43). Mahwah, NJ: Lawrence Erlbaum Associates.

Bright, R. (1996). Grief and powerlessness: Helping people regain control of their lives. London: Jessica Kingsley Publishers.

Calvete, E., Estévez, A., López de Arroyabe, E., \& Ruiz, P. (2005). The Schema Questionnaire-Short form: Structure and relationship with automatic thoughts and sysmtoms of affective disorders. European Journal of Psychological Assessment, 21(2), 90-99. doi:10.1027/1015-5759.21.2.90

Ceballo, R., \& McLoyd, V. C. (2002). Social support and parenting in poor, dangerous neighborhoods. Child Development, 73(4), 1310-1321. doi:10.1111/1467-8624.00473

Chung, K.-S., \& Kim, M. (2016). Anger factors impacting on life satisfaction of mothers with young children in Korea: Does mother's age matter? Personality and Individual Differences, 104, 190-194. doi:10.1016/j.paid.2016.08.010

Clark, D. A., \& Beck, A. T. (2010). Cognitive therapy of anxiety disorders: Science and practice. New York: Guilford Press.

Crnic, K. A., Gaze, C., \& Hoffman, C. (2005). Cumulative parenting stress across the preschool period: Relations to maternal parenting and child behavior at age 5. Infant and Child Development, 14(2), 117-132. doi:10.1002/icd.384

Crnic, K. A., \& Greenberg, M. T. (1990). Minor parenting stress with young children. Child Development, 61(5), 1628-
1637. doi: $10.2307 / 1130770$

Darwin, C. (1965). The expression of emotions in man and animals. IL: University of Chicago Press.

De Jong, J. T. V. M., \& van Ommeren, M. (2002). Toward a culture-informed epidemiology: Combining qualitative and quantitative research in transcultural contexts. Transcultural Psychiatry, 39(4), 422-433. doi:10.1177/136346150203900402

Denham, S. A., Workman, E., Cole, P. M., Weissbrod, C., Kendziora, K. T., \& Zahn-Waxler, C. (2000). Prediction of externalizing behavior problems from early to middle childhood: The role of parental socialization and emotion expression. Development and Psychopathology, 12(1), 23-45. doi:10.1017/s0954579400001024

Diamond, E. L. (1982). The role of anger and hostility in essential hypertension and coronary disease. Psychological Bulletin, 92(2), 410-433. doi:10.1037/0033-2909.92.2.410

Huth-Bocks, A. C., Guyon-Harris, K., Calvert, M., Scott, S., \& Ahlfs-Dunn, S. (2016). The caregiving helplessness questionnaire: Evidence for validity and utility with mothers of infants. Infant Mental Health Journal, 37(3), 208-221. doi:10.1002/imhj.21559

Kang, E. H. (2015). Study on time use pattern of married women on childcare and housework in regards to the number and age of children (Unpublished master thesis). Seoul National University, Seoul, Korea.

Lavee, Y., Sharlin, S., \& Katz, R. (1996). The effects of parenting stress on marital quality: An integrated motherfather model. Journal of Family Issues, 17, 114-135. doi:10.1177/019251396017001007

Lazarus, R. S., \& Averill, J. R. (1972). Emotion and cognition: With special reference to anxiety. In C. D. Spielberger (Ed.), Anxiety: Current trends in theory and research (Vol. 2, pp. 241-290). New York: Academic Press.

Plate, R. C., Bloomberg, Z., Bolt, D. M., Bechner, A. M., Roeber, B. J., \& Pollak, S. D. (2019). Abused children experience high anger exposure. Frontiers in Psychology, 10(440). doi:10.3389/fpsyg.2019.00440

Repetti, R. L., Matthews, K. A., \& Waldron, I. (1989). Employment and women's health: Effects of paid employment on women's mental and physical health. American Psychologist, 44(11), 1394-1401. doi:10.1037/0003-066X.44.11.1394

Rezendes, D. L., \& Scarpa, A. (2011). Associations between parental anxiety/depression and child behavior problems related to autism spectrum disorders: The roles of parenting stress and parenting self-efficacy. Autism Research and Treatment, 2011(395190), 1-10. doi:10.1155/2011/395190

Rodriguez, C., \& Green, A. J. (1997). Parenting stress and anger expression as predictors of child abuse potential. Child Abuse \& Neglect, 21(4), 367-377. doi:10.1016/S01452134(96)00177-9 
Ross, C. E., \& van Willigen, M. (1996). Gender, parenthood, and anger. Journal of Marriage and Family, 58(3), 572-584. doi:10.2307/353718

Spielberger, C. D. (1972). Anxiety as an emotional state. In C. D. Spielberger (Ed.), Anxiety: Current trends in theory and research (Vol. 1, pp.24-49). New York: Academic Press.

Spielberger, C. D., Jacobs, G., Russell, S., \& Crane, R. S. (1983). Assessment of anger: The State-Trait Anger Scale. In J. N. Butcher \& C. D. Spielberger (Eds.), Advances in personality assessment (Vol. 2, pp.159-187). Hillsdale, NJ: Lawrence Erlbaum Association.

Tavris, C. (1982). Anger: The misunderstood emotion. New York: Simon \& Schuster.

Wu, C., \& Chao, R. K. (2017). Parent-adolescent relationship among Chinese immigrant families: An indigenous concept of Qin. Asian American Journal of Psychology, 8(4), 323338. doi:10.1037/aap0000092

Zahn-Waxler, C., Ianotti, R. J., Cummings, E. M., \& Denham, S. (1990). Antecedents of problem behaviors in children of depressed mothers. Development and Psychopathology, 2(3), 271-291. doi:10.1017/S0954579400000778

\section{In Korean}

Byeon, S. (2010). A study on the relationship of the number of child to maternal attitude and maternal efficacy (Master's thesis). Retrieved from http://www.riss.kr/link?id=T12341624

Choi, E. A. (2014). The process of meaning construction on children's happiness by mothers with young children (Doctoral dissertation). Retrieved from http://www.riss.kr/ link?id=T13553205.

Choi, S. (1994). Hangukineau simjung simlihahk [한국인의 심정 심리학]. Chung-Ang Journal of Social Sciences, 7(1), 213237.

Chung, J.-N. (2016). A study on the factors related parenting stress of employed and unemployed mothers with preschoolers. Korean Journal of Human Ecology, 25(6), 665-679. doi:10.5934/kjhe.2016.25.6.665

Chung, K.-S. (2019). The effects of parenting competence and anxiety on anger of mothers with young children. The Journal of Eco Early Childhood Education \& Care, 18(1), 179-206. doi:10.30761/ecoece.2019.18.1.179

Chung, K.-S., Cha, J. R., \& Kim, M. N. (2019). The mediating effects of concern: The relationship between parenting competence and anger expression among mothers with young children. Korean Journal of Childcare and Education, 15(2), 37-57. doi:10.14698/jkcce.2019.15.02.037

Chung, K.-S., \& Kim, J. Y. (2014). Parenting experiences and expectations of mothers with preschoolers. Journal of Lifespan Studies, 4(2), 17-39.
Chung, K.-S., Son, H.-H., Ryu, S.-M., \& Cha, J.-R. (2016). A study on the development of the parent anger scale for mothers with preschool and elementary school children. The Journal of Korea Open Association for Early Childhood Education, 21(1), 515-539. doi:10.20437/KOAECE21-1-22

Jeong, E.-A., \& Kim, E.-Y. (2014). Parenting stress among employed mother with children and family functioning and work intention: Focusing on the policies for work-family balancing. Korean Journal of Local Government \& Administration Studies, 28(2), 239-264.

Kang, M. (2017). Becoming an elementary school parent: Focusing on the experiences of middle-class mothers in Seoul whose children make good grades (Master's thesis). Retrieved from http:// www.riss.kr/link?id=T14619249.

Kim, D.-S. (2017). Identity and role of elementary education in the fourth industrial revolution era. The Journal of Korean Educational Idea, 31(4), 23-45. doi:10.17283/jkedi.2017.31.4.23

Kim, E.-K., \& Koh, C. K. (2016). The relation of parenting stress, anger, and somatization symptom of mothers. The Korean Journal of Stress Research, 24(3), 151-160. doi:10.17547/ kjsr.2016.24.3.151

Kim, G. H. (2011). The effects of employed and unemployed mother's parenting efficacy and parental role satisfaction on life-satisfaction. Family and Environment Research, 49(5), 49-57. doi:10.6115/khea.2011.49.5.049

Kim, J.-H., \& Moon, H.-J. (2006). Variables affecting public and private kindergarten teachers' commitment to their institutions. Journal of Early Childhood Education, 26(5), 307-332.

Kim, K.-W., Doh, H.-S., Kim, S.-W., \& Rhee, S.-H. (2010). The relationship between maternal employment, maternal job characteristics, spousal support, and parenting stress. Korean Journal of Child Studies, 31(5), 101-113.

Kim, M. S., \& Moon, H. J. (2005). Relationship between parenting stress, parenting efficacy on parenting behaviors in mothers with young children. Journal of the Korean Home Economics Association, 43(8), 25-35.

Kwon, M. K. (2011). Parenting stress and related factors of employed and non-employed mothers with infants. Journal of Korean Child Care and Education, 7(2), 19-41.

Lee, M., \& Shin, Y. (2013). Effects of employed and unemployed mother's role, child temperament, and environmental support on early childhood development. Korean Journal of Child Care and Education Policy, 7(1), 1-20.

Park, H., \& Park, J. (2015). The effects of parental psychological control and self-efficacy on social anxiety in middle-school boys and girls. Journal of Future Oriented Youth Society, 12(3), 21-39.

Park, H. J. (2017). The effects of parental anger on young children's resilience (Master's thesis). Retreived from http://www.riss. 
$\mathrm{kr} /$ link?id=T14587377

Park, H. K. (2009). Competition over children's education and the 'full-time mother' identity in Korean middle-class families. Journal of Korean Women's Studies, 25(3), 5-33.

Park, S.-S. (2004). A study on the parenting stress of the dual-earner mothers and fathers (Master's thesis). Retrieved from http:// www.riss.kr/link?id=T9832088

Shin, Y., Kee, J., Woo, S., \& Yoon, J. (2014). Jeochulsan jeongchaeg hwagdae-e ttaleun janyeo yang-yug haengtae byeonhwa bunseog [저출산 정책 확대에 따른 자녀 양육 행태 변화 분석]. Rerieved from Korea Institute for Health and Social Affairs website: https://www.kihasa.re.kr

Sohn, S. M. (2012). Parenting stress and related factors of employed and non-employed mothers with infants. Journal of Future Early Childhood Education, 19(1), 331-357.

Statistics Korea. (2019). 2018nyeon ingudonghyangjosa chulsaeng. samangtonggye jamjeong gyeolgwa [2018년 인구동향조사 출생.사망통계 잠정 결과] Retrieved from Statistics Korea website: http://kostat.go.kr
We, J.-H., \& Chae, K.-M. (2015). Development and validation of Parenting Anxiety Scale. The Korean Journal of Clinical Psychology, 34(4), 945-970. doi:10.15842/kjcp.2015.34.4.005

Yoon, J.-W., Hwang, R. L., \& Cho, H. H. (2009). Parenting stress and related factors of employed and non-employed mothers with preschool children. Korean Journal of Women Health Nursing, 15(4), 294-302. doi:10.4069/kjwhn.2009.15.4.294

\section{ORCID}

$\begin{array}{ll}\text { Kai Sook Chung } & \text { http://orcid.org/0000-0003-4472-824X } \\ \text { Mina Kim } & \text { http://orcid.org/0000-0002-0151-3903 }\end{array}$

Received June 30, 2020

Revision received August 26, 2020

Accepted September 16, 2020 